\title{
Abordaje percutáneo transgástrico en la disrupción ductal del páncreas: serie de casos
}

\section{Percutaneous transgastric approach in pancreatic ductal disruption: Case series}

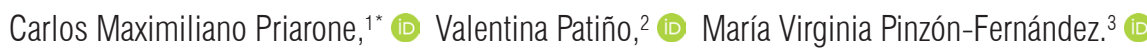

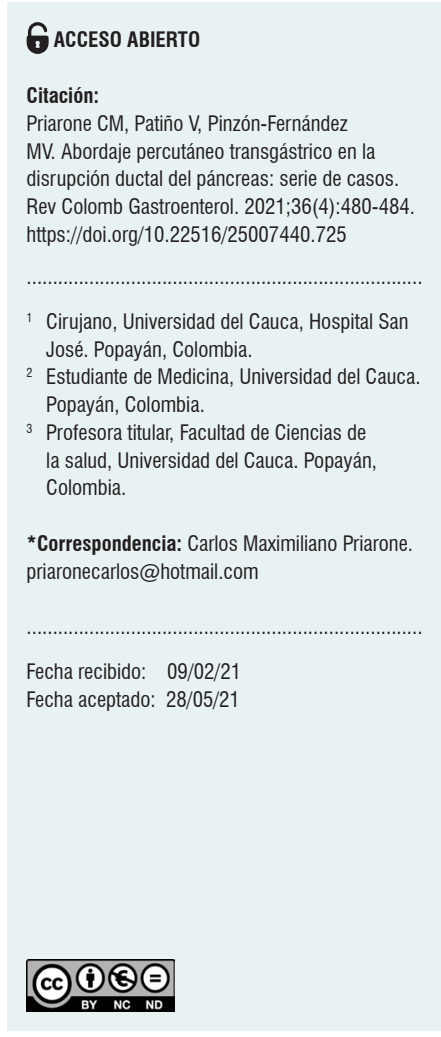

\begin{abstract}
Resumen
Se presentan 6 pacientes con disrupción ductal del páncreas (DDP) tratados mediante drenaje percutáneo guiado por imágenes con apoyo de la endoscopia. Inicialmente, los pacientes presentaron necrosis pancreática infectada tratada con drenaje percutáneo transgástrico y luego de resolver la infección desarrollaron DDP. En el estudio por imágenes se diagnosticaron 4 pacientes con disrupción ductal parcial y 2 con disrupción total, a quienes se les indicó la colocación de una prótesis transgástrica percutánea para lograr el drenaje del líquido pancreático hacia el estómago. La duración promedio de la prótesis fue de 183 días; no hubo casos de mortalidad. Se pudo evidenciar que esta opción terapéutica mínimamente invasiva tiene baja morbimortalidad, por lo que se plantea el abordaje percutáneo transgástrico apoyado por la endoscopia como posible tratamiento de la DDP.
\end{abstract}

\section{Palabras clave}

Pancreatitis aguda necrotizante, procedimientos quirúrgicos mínimamente invasivos, ducto pancreático, pseudoquiste pancreático, fístula pancreática.

\begin{abstract}
A total of six patients with pancreatic ductal disruption (PDD), treated with image-guided endoscopy percutaneous drainage were enrolled. Initially, patients had infected pancreatic necrosis, treated with transgastric percutaneous drainage, and after the infection was controlled, they developed PDD. In the imaging study process, four patients were diagnosed with partial duct disruption and two patients with complete duct disruption. In both cases the placement of a percutaneous transgastric prosthesis to drain the pancreatic fluids to the stomach was indicated. The prosthesis remained 183 days on average and there were no mortality cases. This therapeutic minimally invasive alternative has low rates of morbimortality and thus, the endoscopy percutaneous transgastric approach is considered as a viable treatment in PPD.
\end{abstract}

\section{Keywords}

Acute necrotizing pancreatitis, minimally invasive surgical procedures, pancreatic duct, pancreatic pseudocyst, pancreatic fistula.

\section{INTRODUCCIÓN}

La disrupción ductal del páncreas (DDP) se define como la destrucción parcial o total del ducto pancreático principal, generalmente en el cuello o cuerpo del páncreas, secundaria a necrosis pancreática en el contexto de pancreatitis grave. Debido a que el segmento comprometido continúa con su función exocrina, se crean colecciones intra- o peripancreáticas, fístulas pancreáticas externas (FPE) o débito persistente de líquido pancreático a través del catéter quirúrgico o percutáneo.

En el caso de la disrupción total, la posibilidad de recidiva de las colecciones después del tratamiento endoscópico o percutáneo es mayor, y algunas veces es necesaria 
la resección del segmento pancreático afectado, como tratamiento definitivo. Ante la complejidad de esta patología, se plantean diferentes opciones terapéuticas sin haber definido hasta ahora un estándar de oro ${ }^{(1)}$. El desarrollo de una DDP también se relaciona con procedimientos quirúrgicos traumáticos como necrosectomía endoscópica o quirúrgica $^{(2,3)}$, las cuales son utilizadas para remover la necrosis pancreática infectada (NPI).

El diagnóstico imagenológico de la DDP se realiza mediante pancreatografía endoscópica, resonancia o tomografía. Las complicaciones que pueden desarrollarse luego de una DDP podrían ser colecciones líquidas pancreáticas recurrentes, ascitis, formación de fístulas, pseudoaneurisma, diabetes y deficiencia exocrina ${ }^{(4,5)}$.

El objetivo de este trabajo es describir y analizar los resultados del abordaje percutáneo-endoscópico en una serie de 6 pacientes con DDP.

\section{PRESENTACIÓN DE LOS CASOS}

Se presenta una serie de 6 pacientes atendidos entre 2015 y 2018 diagnosticados con NPI y posteriormente con DDP. Existe evidencia que asocia la necrosis del páncreas con el desarrollo de una DDP aproximadamente en el $20 \%-30 \%$ de los $\operatorname{casos}^{(1,3)}$. Todos los pacientes incluidos en este estudio presentaron una DDP como complicación posterior a la resolución de la NPI.

Para la selección de los pacientes se tuvieron en cuenta los siguientes criterios: haber sido intervenidos por NPI mediante drenaje percutáneo transgástrico, tener diagnóstico de DDP total o parcial, ser mayores de 15 años, haber recibido el alta y seguimiento.

En relación con las características de los pacientes, 5 eran hombres con media de edad de 39 años. Todos los pacientes presentaron NPI como indicación inicial para el drenaje percutáneo. Los abordajes percutáneos para el tratamiento de la NPI fueron el transgástrico, transperitoneal y transretroperitoneal guiado por imágenes, combinándolos dependiendo cada caso clínico (Figura 1 y 2, Tabla 1).

Se utilizaron catéteres multipropósito pigtail, entre 10 y $12 \mathrm{Fr}$. El tiempo promedio de resolución de la NPI fue de 42 días.

En cuanto al diagnóstico de la DDP, 4 fueron mediante tomografía axial computarizada (TAC) y 2 por resonancia magnética (RM). Como resultado, se obtuvieron $4(66,6 \%)$ DDP parciales y $2(33,4 \%)$ totales. Todas las DDP se ubicaron en el cuerpo del páncreas.

Debido a que la DDP no se resolvió con tratamiento médico con octreotida y nutrición parenteral total durante el lapso de 14 días, se colocaron diferentes prótesis utilizando el trayecto del catéter transgástrico. Las prótesis utilizadas fueron: 2 stents biliares doble J (33,4 \%), 1 catéter

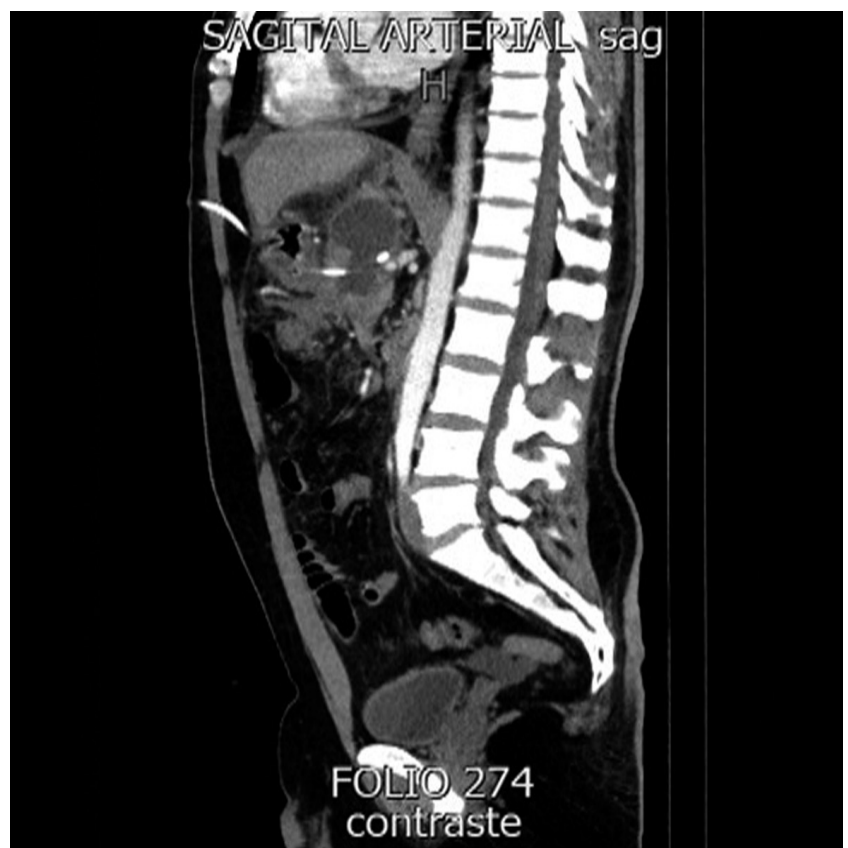

Figura 1. Drenaje transgástrico (corte sagital).

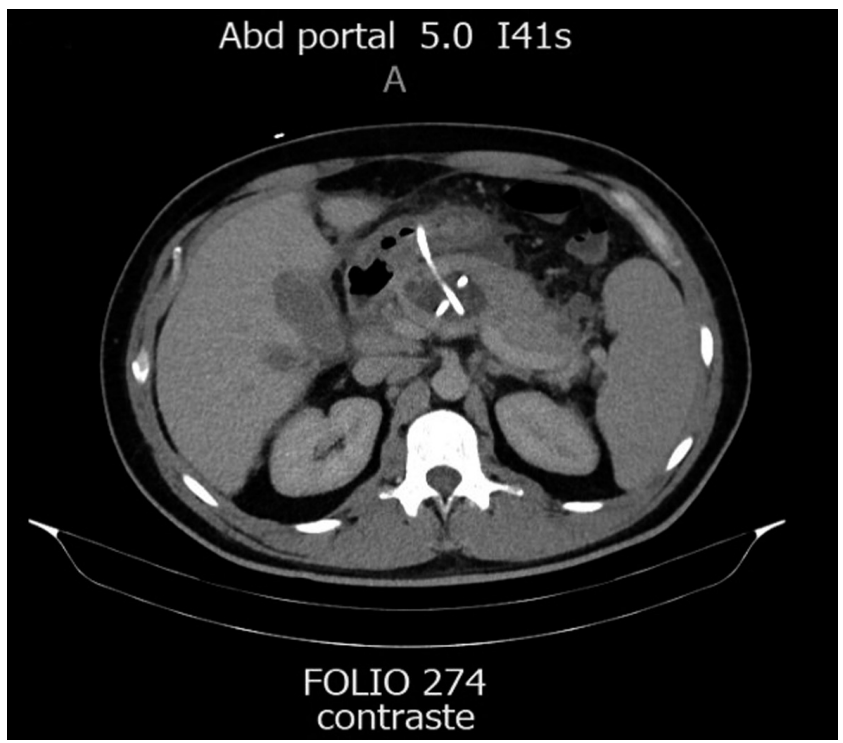

Figura 2. Drenaje transgástrico.

doble J (16,6 \%), 1 catéter pigtail invertido $(16,6 \%)$ y 2 internalizaciones de catéter transgástrico $(33,4 \%)$. Cabe resaltar que en los 2 casos de DDP total se utilizaron un stent biliar doble J y un catéter doble J urológico.

El abordaje para la colocación de las prótesis de drenaje interno fue percutáneo bajo guía fluoroscópica-endoscópica. La duración promedio de la prótesis fue de 183 días (20-376). Un paciente (16,6 \%) presentó migración de 
Tabla 1. Características de los pacientes con NPI

\begin{tabular}{ccccc} 
Paciente & $\begin{array}{c}\text { Tiempo de intervención desde el inicio } \\
\text { de la pancreatitis (dia) }\end{array}$ & $\begin{array}{c}\text { Procedimientos } \\
\text { percutáneos (n) }\end{array}$ & $\begin{array}{c}\text { Abordajes percutáneos } \\
\text { utilizados (n) }\end{array}$ & Microorganismos aislados \\
\hline 1 & 38 & 8 & $\begin{array}{c}\text { Transgástrico (4) } \\
\text { Retroperitoneal (1) } \\
\text { Transperitoneal (3) }\end{array}$ & $\begin{array}{c}\text { Escherichia coli, Pseudomonas } \\
\text { aeruginosa, Enterobacter cloacae, } \\
\text { Proteus mirabilis }\end{array}$ \\
\hline 2 & 20 & 2 & $\begin{array}{c}\text { Transgástrico (1) } \\
\text { Retroperitoneal (1) }\end{array}$ & Citobacter freundii \\
\hline 3 & 18 & 2 & Transgástrico (2) & Acinetobacter baumanii \\
\hline 5 & 22 & 2 & Transgástrico (2) & Enterobacter aerogenes \\
\hline 6 & 25 & 4 & Transperitoneal (2) & Candida krusei, E. aerogenes, \\
\hline
\end{tabular}

la prótesis con la posterior recidiva de una colección de 2 $\mathrm{cm}$, la cual no requirió tratamiento. La prótesis que migró fue el catéter doble J ureteral. El tiempo de hospitalización promedio fue de 69 días y del de seguimiento, 951 días. No hubo mortalidad (Figura 3).

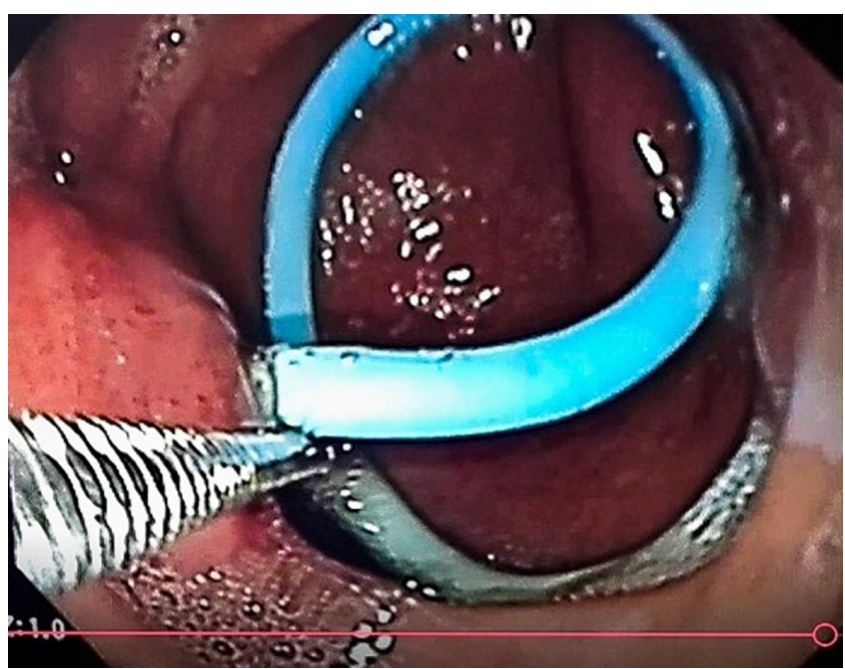

Figura 3. Internalización percutánea de catéter transgástrico.

\section{DISCUSIÓN}

Las complicaciones de la pancreatitis aguda continúan siendo un desafío a pesar del avance en técnicas mínimamente invasivas. Investigaciones muestran un aumento progresivo en la efectividad del abordaje percutáneo y del endoscópico, especialmente después de la llegada de la endosonografía ${ }^{(4,6-8)}$. Ante el diagnóstico de las complicaciones infecciosas tempranas en pancreatitis aguda, como la NPI, el abordaje percutáneo muestra tasas de efectividad que superan el $60 \%{ }^{(9,10)}$ y la necrosectomía endoscópica, como única técnica de tratamiento, podría alcanzar una efectividad del $81 \%{ }^{(11)}$.

Ahora bien, cuando se escoge el drenaje percutáneo, existen 3 vías posibles para la colocación de los diferentes catéteres: la retroperitoneal, peritoneal y la transvisceral (Figura 4).

Por una parte, este abordaje permite la colocación de uno o más catéteres, lo que posibilita la realización de lavados de la necrosis pancreática a través de los mismos. Estos desbridamientos hidráulicos pueden ser realizados en la cama del paciente sin necesidad de anestesia. Por otra parte, el drenaje percutáneo transgástrico es un procedimiento seguro $y$ efectivo, que no ha presentado complicaciones mayores ni mortalidad en nuestra serie, además de que evita procedimientos mayores, abiertos o laparoscópicos para el tratamiento de la NPI. Esto representa beneficios para el paciente, como lo demuestra el estudio PANTER, en el cual la necrosectomía temprana abierta tiene un índice de morbimortalidad mayor que el step-up approach ${ }^{(12)}$.

Actualmente, las prótesis pancreáticas endoscópicas son la primera opción para el tratamiento de la DDP. Sin embargo, el abordaje percutáneo transgástrico podría ser una opción terapéutica, ya que luego de la internalización del catéter, se logra dirigir el líquido pancreático hacia el interior de la cavidad gástrica.

Las guías de pancreatitis aguda no reconocen como más efectiva ninguna de las diferentes vías de abordaje percutá- 

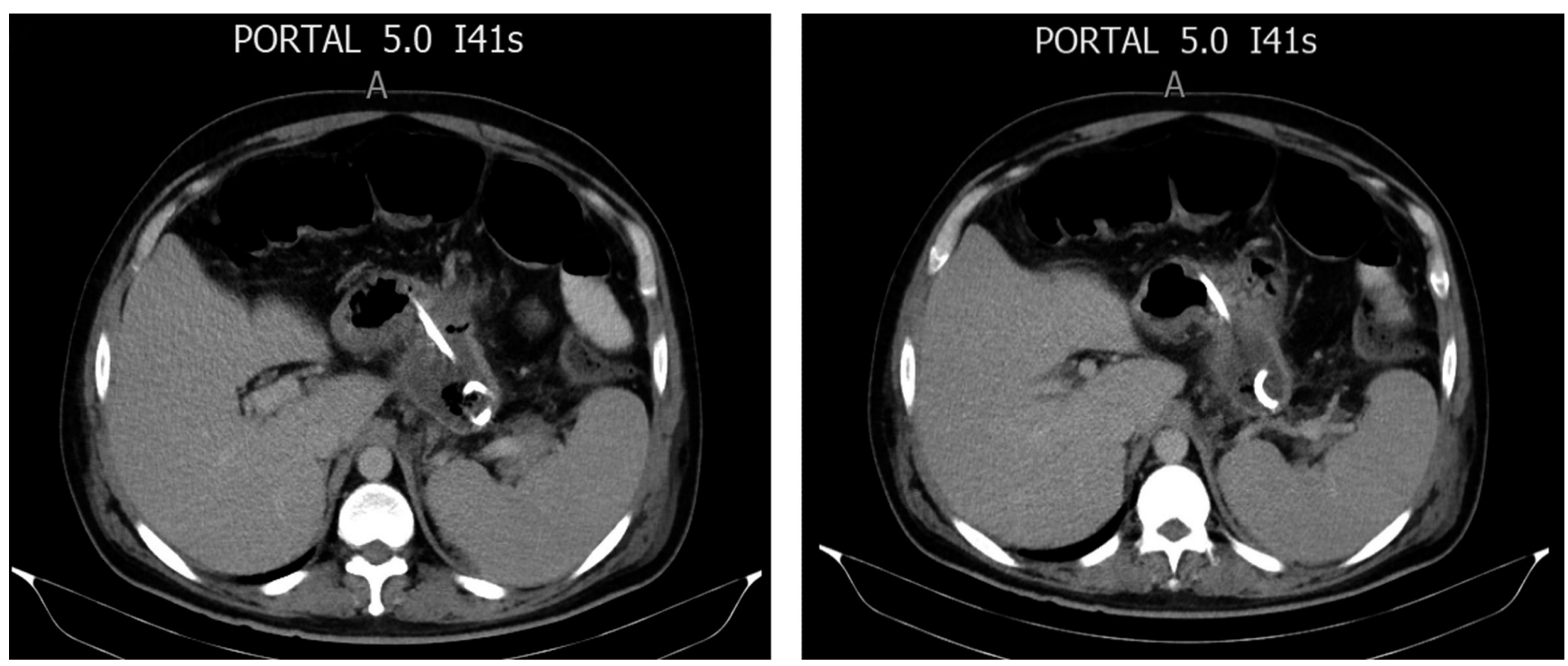

Figura 4. Catéter transgástrico percutáneo internalizado.

neo. No obstante, según esta experiencia, se considera que, ante una complicación de la NPI tratada por vía percutánea, se debería colocar al menos un catéter transgástrico que permita posteriormente la internalización de este, para el tratamiento de una DDP en caso de que esta se presente.

Los dispositivos que se colocaron incluyeron internalización del catéter multipropósito, prótesis como el stent plástico doble J de 7 Fr y catéter doble J urológico (no recomendado debido a la complejidad para su colocación y la posibilidad de migración). La técnica que se recomienda es la internalización del catéter transgástrico bajo guía fluoroscópica y endoscópica, porque técnicamente es sencilla y hay menos posibilidad de migración del catéter en comparación con otras prótesis utilizadas. Probablemente su efectividad sea mayor en casos de disrupción ductal parcial que en la total, como se evidencia en esta serie, en la que se presentó una recidiva en una DDP total como consecuencia de la migración de la prótesis.
Sin embargo, se requieren más trabajos y una mayor cantidad de pacientes para llegar a una conclusión significativa. Se resalta que la mortalidad en el grupo estudiado fue de $0 \%$.

\section{CONCLUSIONES}

El desarrollo de una DDP es un desafío terapéutico. Definir si esta es parcial o total marca una diferencia con respecto a la posibilidad de tratamiento efectivo, de recidiva de colecciones y de la necesidad de una posible resección quirúrgica. El presente estudio permitió evidenciar que la internalización del catéter percutáneo transgástrico es una opción terapéutica para la DDP, con baja morbilidad, sin mortalidad. Sin embargo, se requieren más trabajos y una cantidad mayor de pacientes para poder analizar la morbimortalidad y la efectividad de este tipo de tratamiento a través de un estudio de comparación de técnicas.

\section{REFERENCIAS}

1. Timmerhuis H, Boxhoorn L, Besselink M, Bollen T, Bruno M, Joseph Elmunzer B, Fockens P, Horvath KD, Verdonk RC, van Santvoort HC, Voermans RP . Diagnosis and Treatment of Pancreatic Duct Disruption or Disconnection: an International Expert Survey and Case Vignette Study. Endoscopy. 2020;52(S 01):S124. https://doi.org/10.1055/s-0040-1704381

2. Ramia JM, Fabregat J, Pérez-Miranda M, Figueras J. Síndrome del ducto pancreático desconectado
[Disconnected panreatic duct syndrome]. Cir Esp. 2014;92(1):4-10. https://doi.org/10.1016/j.ciresp.2013.02.024

3. Murage KP, Ball CG, Zyromski NJ, Nakeeb A, Ocampo C, Sandrasegaran K, Howard TJ. Clinical framework to guide operative decision making in disconnected left pancreatic remnant (DLPR) following acute or chronic pancreatitis. Surgery. 2010;148(4):847-56; discussion 856-7. https://doi.org/10.1016/j.surg.2010.07.039 
4. Varadarajulu S, Wilcox CM. Endoscopic placement of permanent indwelling transmural stents in disconnected pancreatic duct syndrome: does benefit outweigh the risks? Gastrointest Endosc. 2011;74(6):1408-12. doi: 10.1016/j. gie.2011.07.049. https://doi.org/10.1016/j.gie.2011.07.049

5. Veillette G, Dominguez I, Ferrone C, Thayer SP, McGrath D, Warshaw AL, Fernández-del Castillo C. Implications and management of pancreatic fistulas following pancreaticoduodenectomy: the Massachusetts General Hospital experience. Arch Surg. 2008;143(5):476-81. https://doi.org/10.1001/archsurg.143.5.476

6. Rana SS, Sharma R, Sharma V, Chhabra P, Gupta R, Bhasin DK. Prevention of recurrence of fluid collections in walled off pancreatic necrosis and disconnected pancreatic duct syndrome: Comparative study of one versus two long term transmural stents. Pancreatology. 2016;16(4):687-8. https://doi.org/10.1016/j.pan.2016.05.009

7. Irani S, Gluck M, Ross A, Gan SI, Crane R, Brandabur JJ, Hauptmann E, Fotoohi M, Kozarek RA. Resolving external pancreatic fistulas in patients with disconnected pancreatic duct syndrome: using rendezvous techniques to avoid surgery (with video). Gastrointest Endosc. 2012;76(3):58693.e1-3. https://doi.org/10.1016/j.gie.2012.05.006

8. Bang JY, Wilcox CM, Navaneethan U, Hasan MK, Peter S, Christein J, Hawes R, Varadarajulu S. Impact of Disconnected Pancreatic Duct Syndrome on the Endoscopic Management of Pancreatic Fluid Collections.
Ann Surg. 2018;267(3):561-568.

https://doi.org/10.1097/SLA.0000000000002082

9. Mouli VP, Sreenivas V, Garg PK. Efficacy of conservative treatment, without necrosectomy, for infected pancreatic necrosis: a systematic review and meta-analysis. Gastroenterology. 2013;144(2):333-340.e2. https://doi.org/10.1053/j.gastro.2012.10.004

10. van Baal MC, van Santvoort HC, Bollen TL, Bakker OJ, Besselink MG, Gooszen HG; Dutch Pancreatitis Study Group. Systematic review of percutaneous catheter drainage as primary treatment for necrotizing pancreatitis. $\mathrm{Br} \mathrm{J}$ Surg. 2011;98(1):18-27. https://doi.org/10.1002/bjs.7304

11. van Brunschot $S$, Fockens P, Bakker OJ, Besselink MG, Voermans RP, Poley JW, Gooszen HG, Bruno M, van Santvoort HC. Endoscopic transluminal necrosectomy in necrotising pancreatitis: a systematic review. Surg Endosc. 2014;28(5):1425-38. https://doi.org/10.1007/s00464-013-3382-9

12. van Santvoort HC, Besselink MG, Bakker OJ, Hofker HS, Boermeester MA, Dejong CH, van Goor H, Schaapherder AF, van Eijck CH, Bollen TL, van Ramshorst B, Nieuwenhuijs VB, Timmer R, Laméris JS, Kruyt PM, Manusama ER, van der Harst E, van der Schelling GP, Karsten T, Hesselink EJ, van Laarhoven CJ, Rosman C, Bosscha K, de Wit RJ, Houdijk AP, van Leeuwen MS, Buskens E, Gooszen HG; Dutch Pancreatitis Study Group. A step-up approach or open necrosectomy for necrotizing pancreatitis. N Engl J Med. 2010;362(16):1491-502. https://doi.org/10.1056/NEJMoa0908821 